\title{
NUMERICAL METHODS FOR THE LANDAU-LIFSHITZ EQUATION*
}

\author{
WEINAN $\mathrm{E}^{\dagger}$ AND XIAO-PING WANG W $^{\ddagger}$
}

\begin{abstract}
We discuss numerical schemes for various forms of the Landau-Lifshitz equation. A new simple projection method is introduced and is shown to be unconditionally stable. The advantages over other schemes are also demonstrated numerically.
\end{abstract}

Key words. Landau-Lifshitz equation, micromagnetics, numerical methods

AMS subject classifications. 65M12, 65M06, 35Q55, 35Q60

PII. S0036142999352199

1. Introduction. The Landau-Lifshitz equation which describes the evolution of magnetization in continuum ferromagnets plays an important role in the understanding of nonequilibrium magnetism. In this paper, we will discuss various numerical methods for the Landau-Lifshitz equation in the form

$$
m_{t}=-m \times \Delta m-\gamma m \times(m \times \Delta m),
$$

where $m: \Omega \subset \mathbf{R}^{d} \rightarrow S^{2}, \mathrm{~d}=1,2,3, \gamma$ is a damping parameter. The boundary condition is taken to be

$$
\frac{\partial m}{\partial n}=0
$$

on $\Gamma=\partial \Omega$. Equation (1.1) is the result of the Landau-Lifshitz equation

$$
m_{t}=-m \times h-\gamma m \times(m \times h)
$$

after neglecting lower order terms [7], [8]. In (1.3), $h=-\frac{\delta F}{\delta m}$ where the free energy $F$ is given by

$$
F(m)=\int_{\Omega}\left\{\frac{1}{2}|\nabla m|^{2}+\Phi(m)+m \cdot \nabla u\right\} d^{3} x .
$$

Here $\Phi$ is a function of $m$, and $u$ solves

$$
\nabla \cdot\left(\nabla u+m \chi_{\Omega}\right)=0
$$

on $\mathbf{R}^{3}$, with $\chi_{\Omega}$ being the indicator function of $\Omega$.

Although the nonlocal term in (1.4) also presents very important computational issues, in this paper we will concentrate on the questions raised by the leading order term in (1.4), thereby neglecting the last two terms at the right-hand side of (1.4). In this case $h=\Delta m$ and (1.3) reduces to (1.1). The three terms in (1.4) are the

\footnotetext{
*Received by the editors February 16, 1999; accepted for publication (in revised form) May 16, 2000; published electronically November 28, 2000.

http://www.siam.org/journals/sinum/38-5/35219.html

† Courant Institute, New York University, New York, NY 10012 (weinan@cims.nyu.edu). Current address: Department of Mathematics and Program in Applied and Computational Mathematics, Princeton University, Princeton, NJ 08544 (weinan@math.princeton.edu).

${ }^{\ddagger}$ Department of Mathematics, The Hong Kong University of Science and Technology, Clear Water Bay, Kowloon, Hong Kong (mawang@ust.hk).
} 
exchange, anisotropy, and magnetostatic energies, respectively. A term of the form $\int_{\Omega} m \cdot H d^{3} x$ should be added to the right-hand side of (1.4) if there is an external field $H$. It is easy to see that (1.3) can also be written as

$$
m_{t}-\gamma m \times m_{t}=-\left(1+\gamma^{2}\right) m \times h
$$

or

$$
\gamma m_{t}+m \times m_{t}=-\left(1+\gamma^{2}\right) m \times(m \times h) .
$$

Equation (1.6) is sometimes referred to as the Gilbert equation.

The following identities will be useful later:

$$
\begin{gathered}
-m \times(m \times h)=h-(m, h) m, \\
-m \times(m \times \Delta m)=\Delta m+|\nabla m|^{2} m,
\end{gathered}
$$

where we have used the fact that $(m, m)=1$.

Two special cases of (1.1) are of particular interest. They correspond to $\gamma=0$ and $\gamma=+\infty$.

$$
\begin{gathered}
m_{t}=-m \times \Delta m, \\
m_{t}=-m \times(m \times \Delta m),
\end{gathered}
$$

or

$$
m_{t}=\Delta m+|\nabla m|^{2} m
$$

Equation (1.11) is the equation describing the heat flow of harmonic maps to $S^{2}$ [12]. It has been studied extensively in the geometry and geometric analysis literature. In contrast, (1.10) describes the Hamiltonian (or symplectic) flow of harmonic maps to $S^{2}$. At this point, there is much less literature on (1.10). Although the two models (1.11) and (1.12) are mathematically equivalent [4], (1.11) gives many more numerically stable solutions than that of (1.12), as we show in section 2 .

Even though it is generally expected, and for special situations of (1.11) rigorously proved [1], that the solutions of (1.1), (1.10), (1.11) develop finite time singularities, we will restrict our attention to smooth solutions of these equations, leaving the discussions on singular solutions to future publications.

In this paper, various numerical schemes will be discussed. In particular, a simple projection scheme is proposed to solve (1.11) which is implicit and unconditionally stable. Time step size is an important issue for the numerical solution of the Landau-Lifshitz equation since the fastest time scale in the application of (1.3) is on the order of picoseconds. This means that straightforward explicit time stepping procedures will suffer from very severe constraints on the size of the time step. On the other hand, implicit schemes will have to deal with the severe nonlinearity present in the equation in the form of the Lagrange multiplier for the constraint that the length of $m$ is 1 . Here we propose a time stepping method in the form of a projection method that circumvents both problems.

The paper is organized as follows. In section 2, we explain the differences between the performance of numerical schemes for (1.11) and (1.12). Convergence of the spatial discretizations is also proved. In section 3, an implicit projection scheme is proposed and its unconditional stability is proved. Numerical examples are given in section 4 to demonstrate that the performance of the projection scheme is better than most of the other numerical schemes. 
2. Spatial discretizations. Most discussions in this section will be in the setting of semidiscrete schemes, i.e., time will be kept continuous. For simplicity of presentation, we will assume that we are working with a uniform grid $\Omega_{h}$ with size $h$. The numerical results we present in this section are computed with sufficiently small $\Delta t$ that the numerical error from time discretization is basically negligible.

On a regular finite difference grid, there are two obvious ways to discretize (1.1). The first is

$$
\frac{d m_{h}}{d t}=-m_{h} \times \Delta_{h} m_{h}-\gamma m_{h} \times\left(m_{h} \times \Delta_{h} m_{h}\right) .
$$

The second is

$$
\frac{d m_{h}}{d t}=-m_{j} \times \Delta_{h} m_{h}+\gamma\left(\Delta_{h} m_{h}+\left|\nabla m_{h}\right|^{2} m_{h}\right) .
$$

Here $\Delta_{h}$ and $\nabla_{h}$ are the standard discretization of $\Delta$ and $\nabla$, respectively, using centered differences. Other difference approximations can be used. But it suffices to discuss this simplest case.

Both (2.1) and (2.2) provide convergent and second order accurate approximations for smooth solutions of (1.1). This is relatively easy to establish for (2.1).

THEOREM 1. Let $m(x, t) \in L^{\infty}\left([0, T], H^{4}\right)$ be a smooth solution of (1.1) with initial data

$$
m(x, 0)=m_{0}(x)
$$

and let $m_{h}$ be the solution of (2.1) with the same initial data on a uniform grid $\Omega_{h}$. Then we have

$$
\max _{x \in \Omega_{h}}\left|m(x, t)-m_{h}(x, t)\right| \leq c(t) h^{2},
$$

where $c(t)$ depends only on $m$.

The proof of this result will be given at the end of this section.

Even though both (2.1) and (2.2) give second order approximations to (1.11), their actual performance is very different. Note that (2.1) preserves the normalization exactly:

$$
\frac{d}{d t}\left(m_{h}, m_{h}\right)=0
$$

and (2.2) does not. Let us examine the numerical solutions for the heat flow of harmonic maps by comparing the results of the following two schemes:

$$
\frac{d m_{h}}{d t}=-m_{h} \times\left(m_{h} \times \Delta_{h} m_{h}\right)
$$

or

$$
\frac{d m_{h}}{d t}=\Delta_{h} m_{h}+\left|\nabla_{h} m_{h}\right|^{2} m_{h} .
$$

In Figures 1 and 2, we plot the time history of the error computed by these two methods, for the exact solution

$$
m_{e}(x, t)=(\sin x \cos t, \sin x \sin t, \cos x)
$$




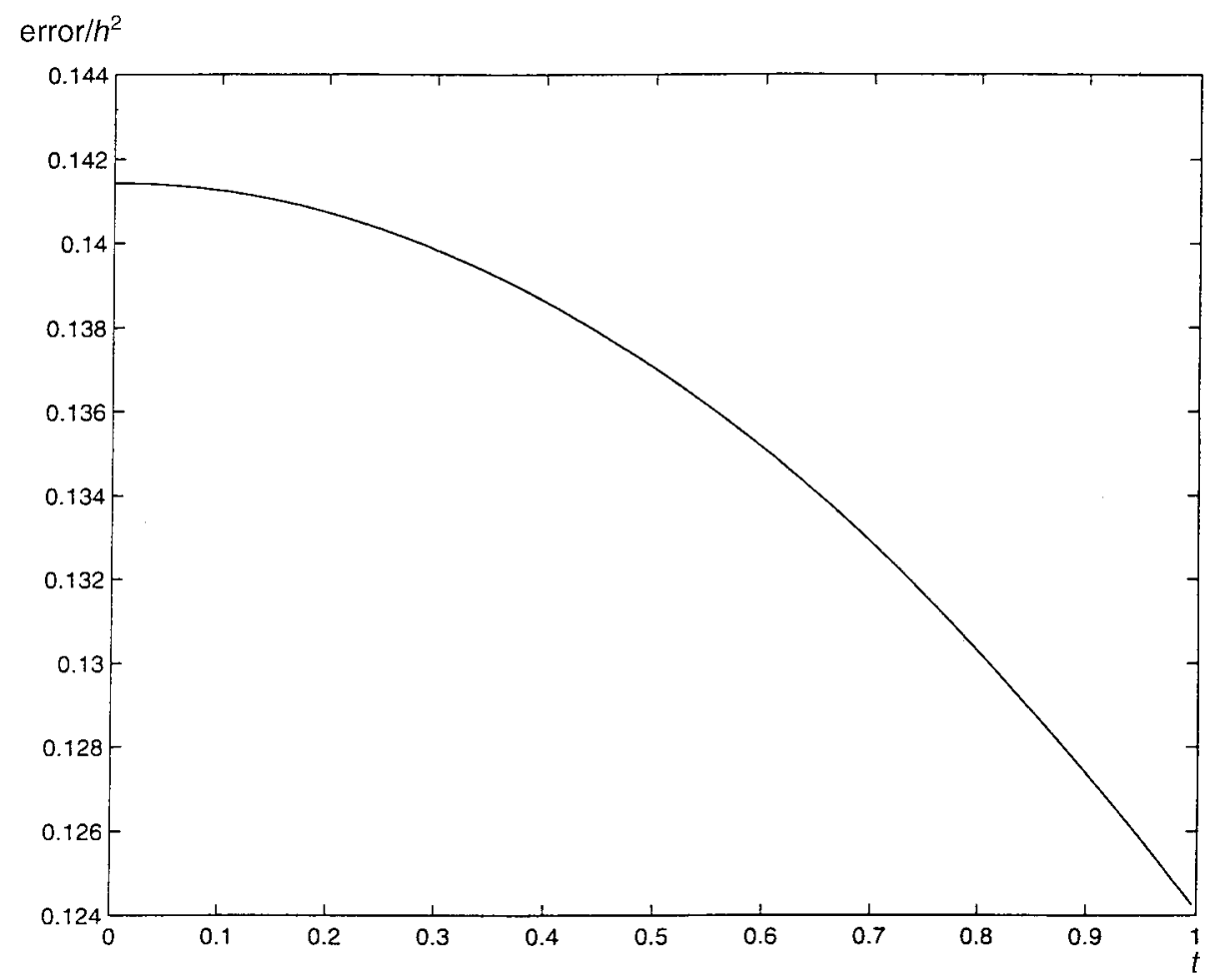

FIG. 1. Time history of the error divided by $h^{2}$ computed using (2.4) with 64 spatial grid points.

on $[0, \pi]$ with Dirichlet boundary condition. For (2.6) to be an exact solution of (1.11), a forcing term $f=\frac{\partial m_{e}}{\partial t}+m_{e} \times\left(m_{e} \times \frac{\partial^{2} m_{e}}{\partial x^{2}}\right)$ has to be added to the right-hand side of (1.11). We can see that while the error for (2.4) remains small, the error for (2.5) grows exponentially fast with time.

The origin of the exponential growth of the error for (2.5) can be understood from the following argument.

Consider the equation

$$
m_{t}=\Delta m+|\nabla m|^{2} m
$$

Let $e=(m, m)-1$. It is easy to see that $e$ satisfies

$$
e_{t}=\Delta e+2|\nabla m|^{2} e .
$$

This shows that if $e$ is not identically zero, then $e$ grows exponentially fast. Since the solutions of (2.5) do not preserve the normalization exactly, we expect $\left(m_{h}, m_{h}\right)-1$ to grow exponentially fast. This means that the error $\left|m-m_{h}\right|$ will exhibit exponential growth.

A simple fix for this problem is to consider instead the following equivalent form of $(2.7)$ :

$$
m_{t}=(m, m) \Delta m+|\nabla m|^{2} m
$$




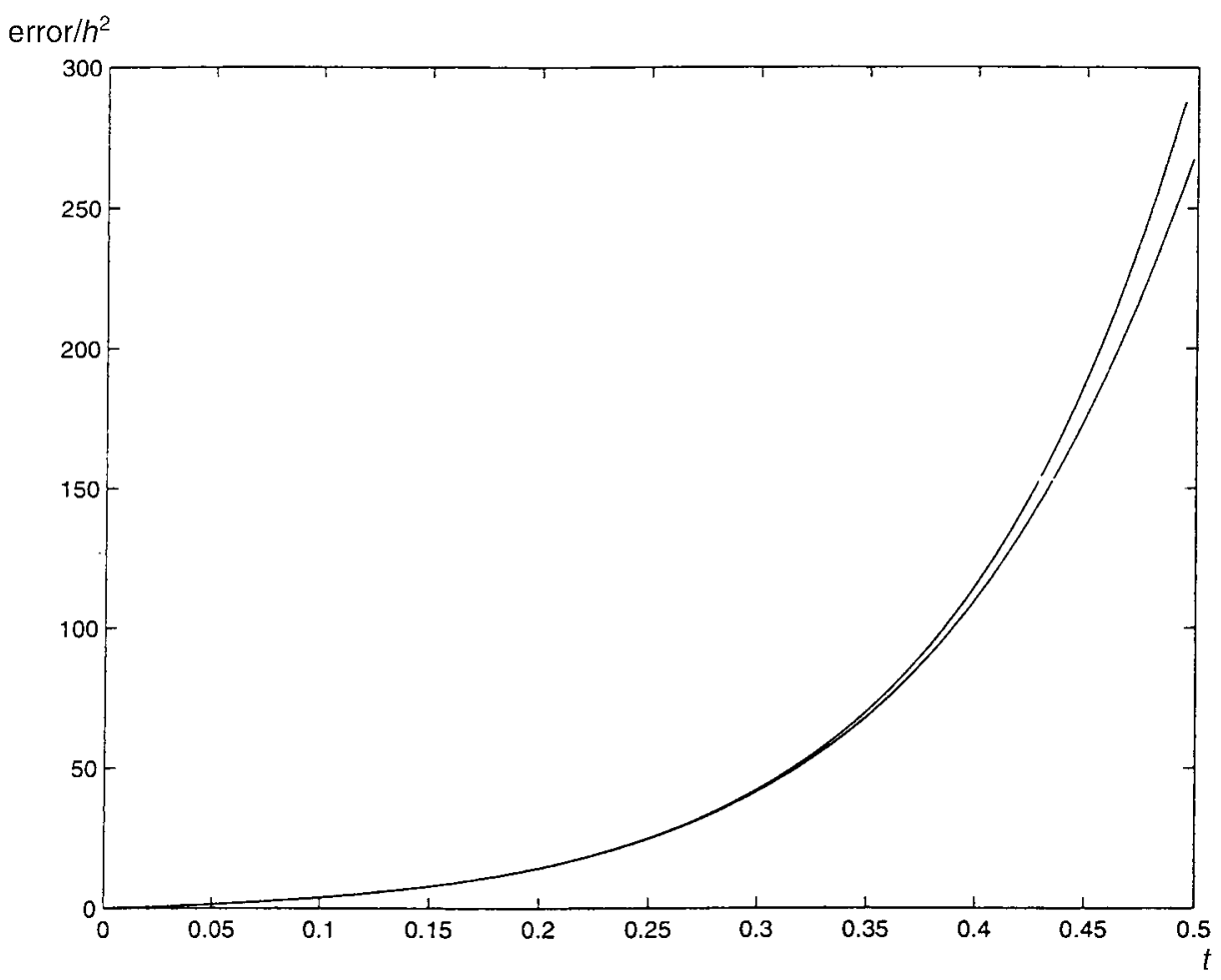

FIG. 2. Time history of the error divided by $h^{2}$ computed using (2.5) with 64 and 128 spatial grid points, respectively.

and replace (2.5) by

$$
\frac{d m_{h}}{d t}=\left(m_{h}, m_{h}\right) \Delta_{h} m+\left|\nabla_{h} m\right|^{2} m_{h} .
$$

For (2.9), (2.8) changes to

$$
e_{t}=(m, m) \Delta e
$$

The term that was responsible for the exponential growth of $e$ in (2.8) is now eliminated.

In Figure 3, we plot the error for the same exact solution as in (2.6) with (2.5) replaced by (2.10). We can see that the exponential growth of the error is now replaced by linear growth.

Proof of Theorem 1.

$$
\begin{gathered}
\frac{\partial m}{\partial t}=-m \times \Delta m-\gamma m \times(m \times \Delta m), \\
\frac{d m_{j}}{d t}=-m_{j} \times \Delta_{h} m_{j}-\gamma m_{j} \times\left(m_{j} \times \Delta_{h} m_{j}\right) .
\end{gathered}
$$

Let $\tilde{m}_{j}(t)=m\left(x_{j}, t\right)$. We have

$$
\frac{d \tilde{m}_{j}}{d t}=-\tilde{m}_{j} \times \Delta_{h} \tilde{m}_{j}-\gamma \tilde{m}_{j} \times\left(\tilde{m}_{j} \times \Delta_{h} \tilde{m}_{j}\right)+O\left(h^{2}\right) .
$$




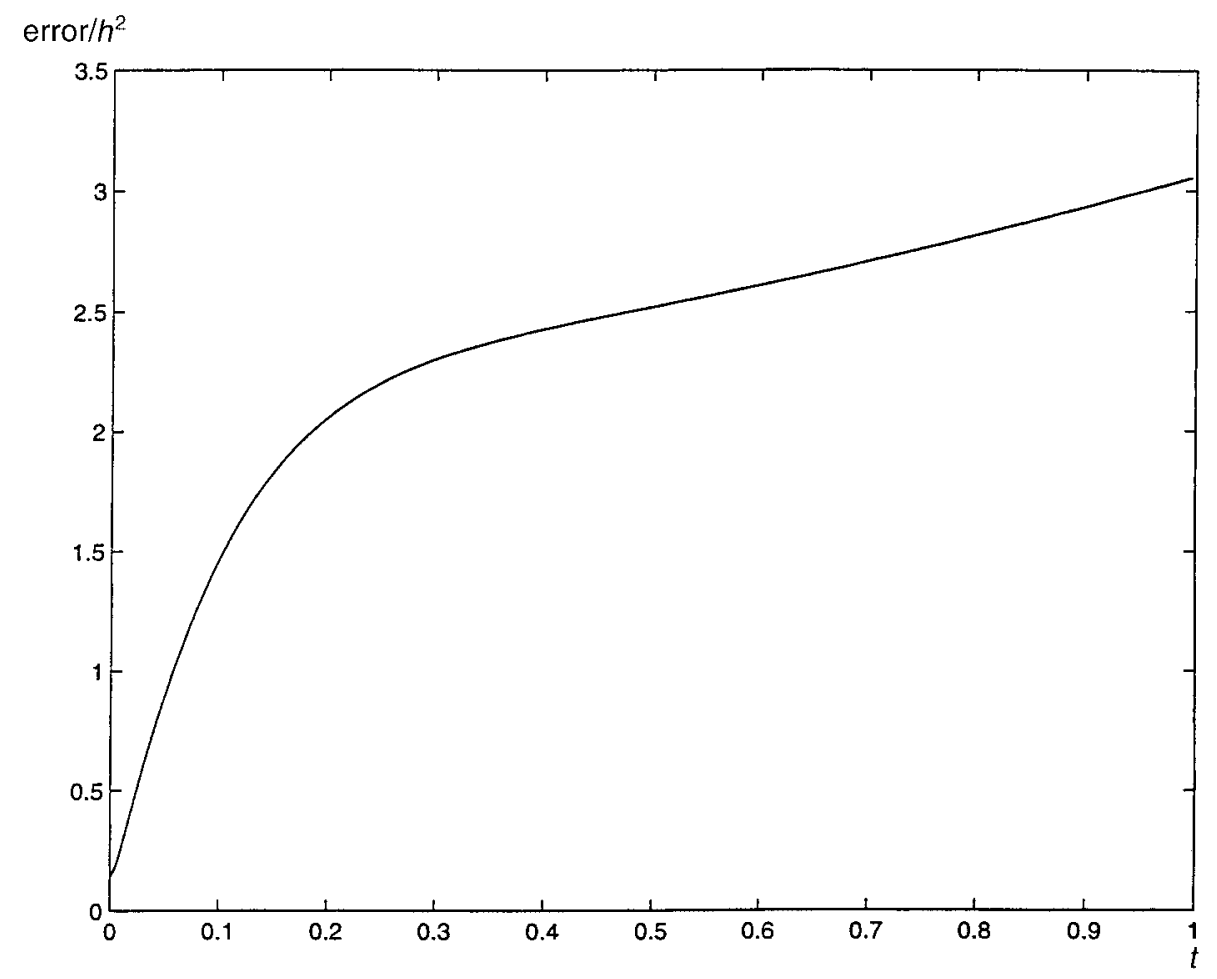

FIG. 3. Time history of the error divided by $h^{2}$ computed using (2.10) with 64 and 128 spatial grid points, respectively.

Denote $e_{j}=m_{j}-\tilde{m}_{j}$; then

$$
\begin{aligned}
\frac{d e_{j}}{d t} & =-m_{j} \times \Delta_{h} e_{j}-e_{j} \times \Delta_{h} \tilde{m}_{j} \\
& -\gamma\left[m_{j} \times\left(m_{j} \times \Delta_{h} e_{j}\right)+m_{j} \times\left(e_{j} \times \Delta_{h} \tilde{m}_{j}\right)\right. \\
& \left.+e_{j} \times\left(\tilde{m}_{j} \times \Delta_{h} \tilde{m}_{j}\right)\right]+O\left(h^{2}\right)
\end{aligned}
$$

and

$$
\begin{aligned}
\sum_{j}\left(\frac{d e_{j}}{d t}, \Delta_{h} e_{j}\right) & =-\sum_{j}\left(e_{j} \times \Delta_{h} \tilde{m}_{j}, \Delta_{h} e_{j}\right) \\
& +\gamma \sum_{j}\left|m_{j} \times \Delta_{h} e_{j}\right|^{2}-\gamma \sum_{j}\left(m_{j} \times\left(e_{j} \times \Delta_{h} \tilde{m}_{j}\right), \Delta_{h} e_{j}\right) \\
& -\gamma \sum_{j}\left(e_{j} \times\left(\tilde{m}_{j} \times \Delta_{h} \tilde{m}_{j}\right), \Delta_{h} e_{j}\right)+\sum_{j}\left(O\left(h^{2}\right), \Delta_{h} e_{j}\right) \\
& =I_{1}+I_{2}+I_{3}+I_{4}
\end{aligned}
$$


and

$$
\begin{aligned}
\frac{1}{2} \frac{d}{d t} \sum_{j}\left(\nabla_{h} e_{j}, \nabla_{h} e_{j}\right) h^{d} & +\gamma \sum_{j}\left|m_{j} \times \Delta_{h} e_{j}\right|^{2} h^{d}=\sum_{j}\left(e_{j} \times \Delta_{h} \tilde{m}_{j}, \Delta_{h} e_{j}\right) h^{d} \\
& +\gamma \sum_{j}\left(m_{j} \times\left(e_{j} \times \Delta_{h} \tilde{m}_{j}\right), \Delta_{h} e_{j}\right) h^{d} \\
& +\gamma \sum_{j}\left(e_{j} \times\left(\tilde{m}_{j} \times \Delta_{h} \tilde{m}_{j}\right), \Delta_{h} e_{j}\right) h^{d}-\sum_{j}\left(O\left(h^{2}\right), \Delta_{h} e_{j}\right) h^{d} \\
& =I_{1}+I_{2}+I_{3}+I_{4}, \\
& \frac{1}{2} \frac{d}{d t} \sum_{j}\left(\nabla_{h} e_{j}, \nabla_{h} e_{j}\right) h^{d} \leq\left|I_{1}\right|+\left|I_{2}\right|+\left|I_{3}\right|+\left|I_{4}\right| .
\end{aligned}
$$

Let us assume for the time being that there exists a $T^{*}<T$, such that (s.t.)

$$
\max _{j}\left|\nabla_{h} m_{j}\right| \leq 2 C_{0}
$$

for $0 \leq t \leq T^{*}$, where $C_{0}$ is chosen so that

$$
C_{0}>\max _{0 \leq t \leq T}|\nabla m|
$$

Then

$$
\begin{gathered}
\left|I_{1}\right| \leq|m|_{3, \infty} \sum_{j}\left|\nabla_{h} e_{j}\right|^{2} h^{d}, \\
\left|I_{2}\right| \leq C_{0}|m|_{3, \infty} \sum_{j}\left|\nabla_{h} e_{j}\right|^{2} h^{d}, \\
\left|I_{3}\right| \leq C_{0}|m|_{3, \infty} \sum_{j}\left|\nabla_{h} e_{j}\right|^{2} h^{d},
\end{gathered}
$$

where $d \leq 3$ is the dimension. Notice that the $O\left(h^{2}\right)$ term is smooth. Summation by parts in $I_{4}$ leads to

$$
\left|I_{4}\right| \leq \sum_{j} O\left(h^{2}\right)\left|\nabla_{h} e_{j}\right| h^{d} \leq O\left(h^{4}\right)+\sum_{j}\left|\nabla_{h} e_{j}\right|^{2} h^{d} .
$$

Equation (2.13) implies that

$$
h^{d} \sum_{j}\left|\nabla_{h} e_{j}\right|^{2} \leq C h^{2},
$$

where $C$ depends on $C_{0}$ and $m$ only.

The assumption (2.14) can be dealt with using Strang's trick. Namely, we construct a correction to the exact solution in the form

$$
\bar{m}(x, t)=m(x, t)+h^{2} m_{1}(x, t)
$$


s.t. $\bar{m}$ satisfies the difference equation with higher order accuracy:

$$
\frac{d \bar{m}_{j}}{d t}=-\bar{m}_{j} \times \Delta_{h} \bar{m}_{j}-\gamma \bar{m}_{j} \times\left(\bar{m}_{j} \times \Delta_{h} \bar{m}_{j}\right)+O\left(h^{4}\right),
$$

where $\bar{m}_{j}(t)=\bar{m}\left(x_{j}, t\right)$. For this purpose, it is necessary and sufficient that $m_{1}$ satisfy

$$
\begin{aligned}
m_{1 t} & =-\frac{1}{12} m \times D^{4} m-m \times \Delta m_{1}-m_{1} \times \Delta m \\
& +\gamma\left[\frac{1}{12} m \times\left(m \times D^{4} m\right)+m \times\left(m \times \Delta m_{1}\right)\right],
\end{aligned}
$$

where

$$
D^{4}=\sum_{i} \partial_{x_{i}}^{4}
$$

The initial condition is $m_{1}(x, 0)=0$ and boundary condition is $\left.\frac{\partial m_{1}}{\partial n}\right|_{\Gamma}=0$. Equation (2.16) is a second order linear parabolic system in $m_{1}$. The existence and uniqueness follows from the general theory in [5].

We have, for $h$ small enough,

$$
\max _{j}\left|\nabla \bar{m}_{j}(t)\right| \leq C_{0}
$$

for $0 \leq t \leq T$. From continuity, there exist $\delta$ small enough, s.t.

$$
\max _{j}\left|\nabla_{h} m_{j}(t)\right| \leq 2 C_{0}
$$

for $0 \leq t \leq \delta$. This means that $T^{*}>\delta$.

Proceeding as in (2.12)-(2.15), we get

$$
h^{d} \sum_{j}\left|\nabla_{h}\left(\bar{m}-m_{j}\right)\right|^{2} \leq C h^{4}
$$

for $0 \leq t \leq \delta$ for some $C$ depending on $C_{0}$ and $\bar{m}$ only. Therefore

$$
\begin{gathered}
\left|\nabla\left(\bar{m}_{j}-m_{j}\right)\right|^{2} \leq C h^{4-d} \leq C h, \\
\left|\nabla m_{j}\right|^{2} \leq 2\left(\left|\nabla \bar{m}_{j}\right|^{2}+C h\right) \leq 3 C_{0}^{2}
\end{gathered}
$$

when $h$ is small enough. This shows that the condition (2.18) will never be violated and $T^{*}$ can be any preset positive value $T$ if $h$ is small enough, depending only on $T$. This completes the proof.

3. Temporal discretization. Again we will look first at the equation for the heat flow of harmonic map, (1.12), and then extend our results to the Landau-Lifshitz equation (1.1).

The main purpose of this section is to construct time discretization procedures that have good stability property. Since we are mainly concerned with temporal discretizations, we will keep the spatial variables continuous. 
3.1. Projection method for the heat flow of harmonic maps. The main idea is to rewrite (1.11) as

$$
m_{t}=\Delta m+\lambda m
$$

and view $\lambda=|\nabla m|^{2}$, or $\lambda=-(m, \Delta m)$ as the Lagrange multiplier for the pointwise constraint $(m, m)=1$.

Projection method is a fractional step procedure in which an intermediate magnetization field, called $m^{*}$, is first computed by disregarding the constraint and the Lagrange multiplier. The intermediate field $m^{*}$ is then projected to $S^{2}$ to obtain the numerical solution at the next time step. The simplest example of such a projection method is the following algorithm:

Knowing $\left\{m^{n}\right\},\left\{m^{n+1}\right\}$ is computed by the following:

Step 1: Solve

$$
\frac{m^{*}-m^{n}}{\Delta t}=\Delta m^{*}
$$

with the boundary condition

$$
\left.\frac{\partial m^{*}}{\partial n}\right|_{\Gamma}=0
$$

Step 2:

$$
m^{n+1}=\frac{m^{*}}{\left|m^{*}\right|}
$$

The simplicity of such a scheme is obvious.

Direct calculation of local truncation error shows that the scheme is of first order accuracy. More generally, consider the equation

$$
m_{t}=h-(m, h) m=-m \times(m \times h) .
$$

The analog of the projection method (3.2)-(3.4) for this equation is

$$
\begin{aligned}
\frac{m^{*}-m^{n}}{\Delta t} & =h\left(t^{n+1}\right), \\
m^{n+1} & =\frac{m^{*}}{\left|m^{*}\right|} .
\end{aligned}
$$

It is easy to check that this method is first order accurate.

To verify the first order convergence of the projection method, we again use the exact solution (2.6) for (1.11) with a forcing term. Table 3.1 gives $\frac{e_{\max }}{\Delta t}$ where $e_{\max }$ is the maximum error.

For this particular example, the scheme provides an accuracy slightly higher than first order.

Next we prove that (3.2)-(3.4) is unconditionally stable and convergent with first order accuracy.

ThEOREM 2. Let $m(x, t) \in L^{\infty}\left([0, T], H^{3}\right)$ be a smooth solution of (1.12) with initial data $m(x, 0)=m_{0}(x)$. Let $m_{\Delta t}$ be the numerical solution of (3.2)-(3.4) with the same initial data. Then we have

$$
\max _{x \in \Omega}\left|m\left(x, t^{n}\right)-m_{\Delta t}\left(x, t^{n}\right)\right| \leq C\left(t^{n}\right) \Delta t,
$$

where $t^{n}=n \Delta t, n=1,2,3, \ldots$ and $C(t)$ depends only on $m$. 
TABLE 3.1

\begin{tabular}{|c|c|c|c|}
\hline & \multicolumn{3}{|c|}{$e_{\max } / \Delta t$} \\
\hline$T$ & $\Delta t=0.01$ & $\Delta t=0.005$ & $\Delta t=0.0025$ \\
\hline 0.2000 & 0.1100 & 0.0878 & 0.0679 \\
0.4000 & 0.1172 & 0.0907 & 0.0684 \\
0.6000 & 0.1197 & 0.0915 & 0.0684 \\
0.8000 & 0.1207 & 0.0918 & 0.0684 \\
1.0000 & 0.1211 & 0.0919 & 0.0684 \\
\hline
\end{tabular}

Proof. We begin by rewriting (3.2)-(3.4) as

$$
m^{n+1}=\frac{(I-\Delta t \Delta)^{-1} m^{n}}{\left|(I-\Delta t \Delta)^{-1} m^{n}\right|}
$$

It is understood in (3.9) that the Neumann boundary condition is imposed when inverting $I-\Delta t \Delta$. Standard local truncation error analysis gives

$$
m\left(x, t^{n+1}\right)=\frac{(I-\Delta t \Delta)^{-1} m\left(x, t^{n}\right)}{\left|(I-\Delta t \Delta)^{-1} m\left(x, t^{n}\right)\right|}+O\left(\Delta t^{2}\right)
$$

if $m$ satisfies (1.12).

In order to deal with the nonlinear recursion relation that arises in the analysis of the error, we will need an adaptation of Strang's trick [11] by constructing a correction of the exact solution of (1.12) which satisfies (3.10) to higher order accuracy. To do this, let

$$
\tilde{m}(x, t)=m(x, t)+\Delta t m_{1}(x, t)+\Delta t^{2} m_{2}(x, t) .
$$

We will choose $m_{1}$ and $m_{2}$ s.t.

$$
\tilde{m}\left(x, t^{n+1}\right)=\frac{(I-\Delta t \Delta)^{-1} \tilde{m}\left(x, t^{n}\right)}{\left|(I-\Delta t \Delta)^{-1} \tilde{m}\left(x, t^{n}\right)\right|}+O\left(\Delta t^{3}\right) .
$$

This is a tedious but straightforward calculation. The key steps of this calculation are summarized below.

$$
\begin{aligned}
\left|(I-\Delta t \Delta)^{-1} \tilde{m}\right|^{2}=1+ & 2 \Delta t\left(m, m_{1}+\Delta m\right) \\
+\Delta t^{2} & \left(2\left(m, m_{2}+\Delta^{2} m+\Delta m_{1}\right)+\left|m_{1}+\Delta m\right|^{2}\right)+O\left(\Delta t^{3}\right), \\
\left|(I-\Delta t \Delta)^{-1} \tilde{m}\right|^{-1} & =1-\Delta t\left(m, m_{1}+\Delta m\right) \\
& -\Delta t^{2}\left(m, m_{2}+\Delta^{2} m+\Delta m_{1}\right)-\frac{\Delta t^{2}}{2}\left|m_{1}+\Delta m\right|^{2} \\
& +\frac{3}{2} \Delta t^{2}\left(m, m_{1}+\Delta m\right)^{2}+O\left(\Delta t^{3}\right),
\end{aligned}
$$

$$
\begin{aligned}
\frac{(I-\Delta t \Delta)^{-1} \tilde{m}}{\left|(I-\Delta t \Delta)^{-1} \tilde{m}\right|} & =m+\Delta t m_{1}+\Delta t^{2} m_{2}+\Delta t \Delta m+\Delta t^{2} \Delta m_{1}+\Delta t^{2} \Delta^{2} m \\
& -\Delta t\left(m, m_{1}+\Delta m\right) m-\Delta t^{2}\left(m, \Delta m_{1}\right) m \\
& -\Delta t^{2}\left(m, m_{1}+\Delta m\right) m_{1}-\Delta t^{2}\left(m, m_{1}+\Delta m\right) \Delta m \\
& -\Delta t^{2}\left[\left(m, m_{2}+\Delta^{2} m\right)+\frac{1}{2}\left|m_{1}+\Delta m\right|^{2}\right. \\
& \left.-\frac{3}{2}\left(m, m_{1}+\Delta m\right)^{2}\right] m+O\left(\Delta t^{3}\right) .
\end{aligned}
$$


From (3.15), we see that in order to satisfy (3.12), $m_{1}$ and $m_{2}$ must obey

$$
\left(m_{1}, m\right)=0
$$

$$
\begin{aligned}
\frac{\partial m_{1}}{\partial t}+\frac{1}{2} \frac{\partial^{2} m}{\partial t^{2}} & =\Delta \tilde{m}_{1}-(m, \Delta m) \tilde{m}_{1} \\
& -\left\{\left(m, \Delta \tilde{m}_{1}\right)+\frac{1}{2}\left|\tilde{m}_{1}\right|^{2}\right. \\
& \left.-\frac{3}{2}(m, \Delta m)^{2}+\left(m, m_{2}\right)\right\} m
\end{aligned}
$$

where $\tilde{m}_{1}=m_{1}+\Delta m$. We can rewrite $(3.17)$ as

$$
\frac{\partial m_{1}}{\partial t}=\Delta m_{1}+a(x, t) m_{1}+b(x, t)-\mu m,
$$

where $a(x, t)=|\nabla m|^{2}, b(x, t)=\Delta^{2} m+|\nabla m|^{2} \Delta m-\frac{1}{2} m_{t t}$ are known functions depending on $m(x, t) . \mu(x, t)$ can be viewed as the Lagrangian multiplier for the constraint (3.16). In Appendix A, we show that with initial and boundary conditions

$$
m_{1}(x, 0)=0,\left.\quad \frac{\partial m_{1}}{\partial n}\right|_{\Gamma}=0
$$

there exists a unique $\mu(x, t)$ s.t. the solution $m_{1}(x, t)$ of the linear equation (3.18) satisfies (3.16). Once $m_{1}$ is determined, $m_{2}$ is chosen so that (3.17) and (3.18) are consistent. This completes the construction of $\tilde{m}$.

Now we can proceed with the error estimates. Let $e^{n}(x)=m^{n}(x)-\tilde{m}\left(x, t^{n}\right), \quad \bar{m}=$ $(I-\Delta t \Delta)^{-1} \tilde{m}\left(t^{n}\right), \quad \tilde{e}=m^{\star}-\bar{m}$. Then from $(3.12)$, we have

$$
e^{n+1}=m^{n+1}-\tilde{m}\left(x, t^{n+1}\right)=\frac{m^{\star}}{\left|m^{\star}\right|}-\frac{\bar{m}}{|\bar{m}|}+O\left(\Delta t^{3}\right) .
$$

Using the elementary inequality

$$
\left|\frac{m^{\star}}{\left|m^{\star}\right|}-\frac{\bar{m}}{|\bar{m}|}\right| \leq \max \left(\frac{1}{\left|m^{\star}\right|} \frac{1}{|\bar{m}|}\right)\left|m^{\star}-\bar{m}\right|,
$$

we obtain

$$
\left|e^{n+1}\right| \leq \max \left(\frac{1}{\left|m^{\star}\right|,|\bar{m}|}\right)|\tilde{e}|+O\left(\Delta t^{3}\right) .
$$

LEMma 1. Assume that

$$
(I-\Delta) u=f
$$

$$
\left.\frac{\partial u}{\partial n}\right|_{\Gamma}=0
$$

where $u=\left(u_{1}, u_{2}, u_{3}\right), \quad f=\left(f_{1}, f_{2}, f_{3}\right)$. Then

$$
\max _{x}|u| \leq \max _{x}|f| .
$$


Proof. A direct computation gives

$$
\Delta|u|=\frac{1}{|u|}\left[(u, \Delta u)+|\nabla u|^{2}-\frac{|(u, \nabla u)|^{2}}{|u|^{2}}\right] .
$$

Therefore

$$
\begin{aligned}
(I-\Delta)|u| & =|u|-\Delta|u| \\
& =\frac{1}{|u|}(u, f)-\frac{1}{|u|}\left(|\nabla u|^{2}-\frac{|(u, \nabla u)|^{2}}{|u|^{2}}\right) \\
& \leq \frac{1}{|u|}(u, f) \leq|f| .
\end{aligned}
$$

We also have $\frac{\partial}{\partial n}|u|=0$ at the boundary. Now (3.25) follows directly from the strong maximum principle.

Continue now with the proof of the theorem. We have from (3.22) and the previous lemma that

$$
\left|e^{n+1}\right| \leq \max \left(\frac{1}{\left|m^{\star}\right|,} \frac{1}{|\bar{m}|}\right)\left|e^{n}\right|+O\left(\Delta t^{3}\right) .
$$

Let $T^{\star}$ be a time (which may depend on $\Delta t$ ) s.t.

$$
\frac{1}{\left|m^{*}\right|} \leq \frac{1}{|\bar{m}|}+\Delta t
$$

for $0 \leq t \leq T^{\star}$. Since $\frac{1}{|\bar{m}|} \leq 1+C \Delta t$ for some constant $C$ which depends only on $t$ and $m$, we get

$$
\left|e^{n+1}\right| \leq(1+C \Delta t)\left|e^{n}\right|+O\left(\Delta t^{3}\right)
$$

for a different $C$. Therefore

$$
\left|e^{n+1}\right| \leq C_{0} \Delta t^{2}
$$

if $n \Delta t \leq T^{\star}$. Here $C_{0}$ does not depend on $\Delta t$.

Now let us estimate $T^{\star}$. Assume that (3.29) holds for $0 \leq t \leq n \Delta t$. Then for $t=(n+1) \Delta t$,

$$
\frac{1}{\left|m^{\star}\right|}-\frac{1}{|\bar{m}|} \leq \frac{\left|m^{\star}-\bar{m}\right|}{\left|m^{\star}\right||\bar{m}|} \leq \frac{C_{o} \Delta t^{2}}{\left|m^{\star}\right||\bar{m}|} \leq \frac{C \Delta t^{2}}{\left|m^{*}\right|} .
$$

Hence

$$
\begin{aligned}
\frac{1}{\left|m^{\star}\right|} & \leq \frac{1}{1-C \Delta t^{2}} \frac{1}{|\bar{m}|} \\
& \leq \frac{1}{|\bar{m}|}+C_{1} \Delta t^{2},
\end{aligned}
$$

where $C_{1}$ does not depend on $\Delta t$. If $\Delta t$ is small enough s.t. $C_{1} \Delta t<1$, we see that (3.29) is still satisfied at $(n+1) \Delta t$. This argument shows that $T^{\star}$ can be any preset positive value $T$ by choosing $\Delta t$ small enough, depending only on $T$. This completes the proof. 
3.2. The second order scheme. Our next task is to look for the second order versions of the projection method. It is easy to check that the two-step method

$$
\begin{gathered}
\frac{m^{\star}-m^{n}}{\Delta t}=\Delta \frac{m^{\star}+m^{n}}{2}, \\
m^{n+1}=\frac{m^{\star}}{\left|m^{\star}\right|}
\end{gathered}
$$

gives only a first order accurate approximation. However, we can add correction terms to (3.34) to achieve the second order accuracy.

We solve the heat flow of harmonic map

$$
m_{t}=\Delta m+|\nabla m|^{2} m
$$

with the following scheme:

$$
\begin{aligned}
& \frac{m^{*}-m^{n}}{\Delta t}=\alpha \Delta m^{*}+f\left(m^{n}\right)+\Delta t g\left(m^{n}\right), \\
& m^{n+1}=\frac{m^{*}}{\left|m^{*}\right|},
\end{aligned}
$$

where $\alpha, f$, and $g$ are to be determined so that the scheme is second order, i.e.,

$$
\begin{aligned}
\left.m\right|_{t=t^{n+1}} & =\left.\frac{(I-\alpha \Delta t \Delta)^{-1}\left(m+\Delta t f+(\Delta t)^{2} g\right)}{\left|(I-\alpha \Delta t \Delta)^{-1}\left(m+\Delta t f+(\Delta t)^{2} g\right)\right|}\right|_{t=t^{n}}+O\left((\Delta t)^{3}\right) \\
& =\left.\frac{H(t)}{|H(t)|}\right|_{t=t^{n}}+O\left((\Delta t)^{3}\right),
\end{aligned}
$$

where

$$
\begin{aligned}
H(t) & =(I-\alpha \Delta t \Delta)^{-1}\left(m(t)+\Delta t f(m(t))+(\Delta t)^{2} g(m(t))\right) \\
& \approx m+\Delta t(f+\alpha \Delta m)+(\Delta t)^{2}\left(g+\alpha \Delta f+\alpha^{2} \Delta^{2} m\right) .
\end{aligned}
$$

Simple calculations (in Appendix B) show that we shall take

$$
f=\frac{1}{2} \Delta m
$$

and

$$
g=\nabla\left(|\nabla m|^{2}\right) \cdot \nabla m,
$$

i.e., $g_{j}=\nabla\left(|\nabla m|^{2}\right) \cdot \nabla m_{j}$ for $j=1,2$ and 3. Therefore, we have a second order scheme

$$
\begin{aligned}
& \frac{m^{*}-m^{n}}{\Delta t}=\frac{1}{2}\left(\Delta m^{*}+\Delta m^{n}\right)+\Delta t \nabla\left(\left|\nabla m^{n}\right|^{2}\right) \cdot \nabla m^{n}, \\
& m^{n+1}=\frac{m^{*}}{\left|m^{*}\right|}
\end{aligned}
$$

Note that (3.41) is no longer unconditionally stable because of the correction term. However, it is easy to see that the CFL condition is $\frac{d t}{d x} \leq C$. For the example (S6) in section $4, C$ is calculated numerically to be 0.509 . 
TABLE 3.2

\begin{tabular}{|c|c|c|c|}
\hline & \multicolumn{3}{|c|}{$e_{\max } / \Delta t$} \\
\hline$T$ & $\Delta t=0.01$ & $\Delta t=0.005$ & $\Delta t=0.0025$ \\
\hline 0.200000 & 3.831069 & 3.179168 & 2.555914 \\
0.400000 & 4.015820 & 3.355095 & 2.747515 \\
0.600000 & 4.230660 & 3.535197 & 2.950982 \\
0.800000 & 4.399771 & 3.680398 & 3.126004 \\
1.000000 & 4.558102 & 3.825170 & 3.287008 \\
\hline
\end{tabular}

3.3. Extension to the Landau-Lifshitz equation. To extend the projection method to the Landau-Lifshitz equation, we will use (1.7). To simplify writing we will omit the coefficients $\gamma$ and $\frac{1+\gamma^{2}}{\gamma}$ and consider

$$
m_{t}+m \times m_{t}=-m \times(m \times \Delta m)=\Delta m+|\nabla m|^{2} m .
$$

The simplest projection scheme for (3.43) is given by the following two-step procedure:

$$
\frac{m^{*}-m^{n}}{\Delta t}+m^{n} \times \frac{m^{*}-m^{n}}{\Delta t}=\Delta m^{*}
$$

with the boundary condition $\left.\frac{\partial m^{*}}{\partial n}\right|_{\Gamma}=0$, and

$$
m^{n+1}=\frac{m^{*}}{\left|m^{*}\right|}
$$

We can formally write (3.44)-(3.45) as

$$
m^{n+1}=\frac{\left(I+m^{n} \times-\Delta t \Delta\right)^{-1} m^{n}}{\left|\left(I+m^{n} \times-\Delta t \Delta\right)^{-1} m^{n}\right|} .
$$

It is easy to see that the scheme is first order accurate. Similar calculations give a second order scheme as follows:

$$
\left\{\begin{array}{l}
\frac{m^{*}-m^{n}}{\Delta t}+m^{n} \times \frac{m^{*}-m^{n}}{\Delta t}=\frac{1}{2}\left(\Delta m^{*}+\Delta m^{n}\right) \\
\quad+(\Delta t)^{2}\left\{\nabla\left(\left|\nabla m^{n}\right|^{2}\right) \cdot \nabla m^{n}+\frac{1}{2}\left|\nabla m^{n}\right|^{2} B \Delta m^{n}\right\} \\
m^{n+1}=\frac{m^{*}}{\left|m^{*}\right|},
\end{array}\right.
$$

where

$$
B=\left(I+m^{n} \times\right)^{-1}-I .
$$

Again, convergence of the scheme (3.44) and (3.45) is verified by computing the exact solution (2.6) for (3.43) with a forcing term. Table 3.2 shows the ratio of the maximum error to $\Delta t$ for different $\Delta t$. A slightly better than first order accuracy is obtained in this case.

4. Comparison of the performances of various numerical schemes. In this section, we present numerical results for (1.11), (1.12), and (2.9) in one dimension. We will compare the results for various schemes. The equations are solved on interval $[0, \pi]$ with initial conditions

$$
m(x, 0)=\left(\begin{array}{c}
\cos (x / 2) \sin (x) \\
\sin (x / 2) \sin (x) \\
\cos (x)
\end{array}\right) .
$$


The "exact" solution is calculated by the fourth order Runge-Kutta scheme in time and the second order center difference with 800 grid points and $\Delta t=10^{-6}$.

We will give numerical results for the following schemes:

(1) Forward Euler for (1.11):

$$
\frac{m^{n+1}-m^{n}}{\Delta t}=-m^{n} \times\left(m^{n} \times \Delta_{h} m^{n}\right) .
$$

(2) Forward Euler for (1.12):

$$
\frac{m^{n+1}-m^{n}}{\Delta t}=\Delta_{h} m^{n}+\left|\nabla_{h} m^{n}\right|^{2} m^{n} .
$$

(3) Forward Euler for (2.9):

$$
\frac{m^{n+1}-m^{n}}{\Delta t}=\left(m^{n}, m^{n}\right) \Delta_{h} m^{n}+\left|\nabla_{h} m^{n}\right|^{2} m^{n} .
$$

(4) Backward Euler for (1.12):

$$
\frac{m^{n+1}-m^{n}}{\Delta t}=\Delta_{h} m^{n+1}+\left|\nabla_{h} m^{n}\right|^{2} m^{n} .
$$

(5) The first order projection method for (1.12):

$$
\left\{\begin{array}{l}
\frac{m^{*}-m^{n}}{\Delta t}=\Delta_{h} m^{*} \\
m^{n+1}=\frac{m^{*}}{\left|m^{*}\right|}
\end{array}\right.
$$

(6) The second order projection method for (1.12):

$$
\left\{\begin{array}{l}
\frac{m^{*}-m^{n}}{\Delta t}=\Delta_{h} \frac{m^{*}+m^{n}}{2}+\Delta t \nabla_{h}\left(\left|\nabla_{h} m^{n}\right|^{2}\right) \nabla_{h} m^{n}, \\
m^{n+1}=\frac{m^{*}}{\left|m^{*}\right|}
\end{array}\right.
$$

Table 4.1 shows the error for these schemes with $\Delta t=0.0001, \Delta x=\pi / 200$. For (S2), the error grows too fast. Stable results for (S3), (S4), (S5) can also be obtained with $\Delta t$ as large as 0.1 for the $\Delta x$ given above. For ( $\mathrm{S} 6), \Delta t$ is slightly restrictive to 0.08 . However, (S1) can be run only for $\Delta t=0.0001$ due to CFL condition.

The results show that for the first order schemes, the accuracy for (S1) and (S5) are comparable and both are much better than (S2), (S3), and (S4). However, (S5) is unconditionally stable (although more expensive) while (S1) is restricted by the CFL condition. Therefore the projection scheme is a better scheme not only for its simplicity but also for its stability and accuracy.

5. Appendix A: A proof of existence of the Lagrangian multiplier. Let $G(x, y, t)$ be the Green's function for the equation

$$
u_{t}=\Delta u+a(x, t) u
$$

with boundary condition

$$
\left.\frac{\partial u}{\partial n}\right|_{\Gamma}=0
$$


TABLE 4.1

\begin{tabular}{|c|c|c|c|c|c|c|}
\hline $\mathrm{T}$ & S1 & S2 & S3 & S4 & S5 & S6 \\
\hline 0.5 & $0.75291 \mathrm{E}-05$ & & $0.60626 \mathrm{E}-01$ & $0.48056 \mathrm{E}-04$ & $0.34438 \mathrm{E}-04$ & $0.93681 \mathrm{E}-05$ \\
1.0 & $0.63458 \mathrm{E}-05$ & & $0.80183 \mathrm{E}-01$ & $0.11777 \mathrm{E}-03$ & $0.14998 \mathrm{E}-04$ & $0.41371 \mathrm{E}-05$ \\
1.5 & $0.59942 \mathrm{E}-05$ & & $0.11340 \mathrm{E}+00$ & $0.24742 \mathrm{E}-03$ & $0.51972 \mathrm{E}-05$ & $0.14723 \mathrm{E}-05$ \\
2.0 & $0.59510 \mathrm{E}-05$ & & $0.12362 \mathrm{E}+00$ & $0.46078 \mathrm{E}-03$ & $0.24537 \mathrm{E}-05$ & $0.67426 \mathrm{E}-06$ \\
2.5 & $0.59471 \mathrm{E}-05$ & & $0.12292 \mathrm{E}+00$ & $0.81217 \mathrm{E}-03$ & $0.24376 \mathrm{E}-05$ & $0.55257 \mathrm{E}-06$ \\
3.0 & $0.59468 \mathrm{E}-05$ & & $0.12275 \mathrm{E}+00$ & $0.13906 \mathrm{E}-02$ & $0.24354 \mathrm{E}-05$ & $0.54592 \mathrm{E}-06$ \\
3.5 & $0.59468 \mathrm{E}-05$ & & $0.12273 \mathrm{E}+00$ & $0.23421 \mathrm{E}-02$ & $0.24350 \mathrm{E}-05$ & $0.54819 \mathrm{E}-06$ \\
4.0 & $0.59468 \mathrm{E}-05$ & & $0.12273 \mathrm{E}+00$ & $0.39047 \mathrm{E}-02$ & $0.24350 \mathrm{E}-05$ & $0.55060 \mathrm{E}-06$ \\
4.5 & $0.59468 \mathrm{E}-05$ & & $0.12273 \mathrm{E}+00$ & $0.64647 \mathrm{E}-02$ & $0.24350 \mathrm{E}-05$ & $0.55304 \mathrm{E}-06$ \\
5.0 & $0.59468 \mathrm{E}-05$ & & $0.12273 \mathrm{E}+00$ & $0.10642 \mathrm{E}-01$ & $0.24350 \mathrm{E}-05$ & $0.55573 \mathrm{E}-06$ \\
\hline CPU(seconds) & 22.64 & & 28.66 & 44.45 & 40.60 & 56.21 \\
\hline
\end{tabular}

the solution of (3.18) is given by

$$
m_{1}(x, t)=\int_{0}^{t} \int_{\Omega} G(x, y, t-s)(b(y, s)-\mu(y, s) m(y, s)) d y d s .
$$

If we require

$$
\left(m_{1}, m\right)=0
$$

then we have

$$
\begin{aligned}
& \int_{0}^{t} \int_{\Omega} G(x, y, t-s) \mu(y, s)(m(y, s), m(x, t)) d y d s \\
= & \int_{0}^{t} \int_{\Omega} G(x, y, t-s)(b(y, s), m(x, t)) d y d s=f(x, t) .
\end{aligned}
$$

Differentiating with respect to $t$, we have

$$
\begin{aligned}
\mu(x, t) & +\int_{0}^{t} \int_{\Omega} G_{t}(x, y, t-s) \mu(y, s)(m(y, s), m(x, t)) d y d s \\
& +\int_{0}^{t} \int_{\Omega} G(x, y, t-s) \mu(y, s)\left(m(y, s), m_{t}(x, t)\right) d y d s \\
& =f_{t}(x, t) .
\end{aligned}
$$

Applying the Laplacian to (5.1), we have

$$
\begin{aligned}
& \int_{0}^{t} \int_{\Omega} \Delta G(x, y, t-s) \mu(y, s)(m(y, s), m(x, t)) d y d s \\
+ & 2 \sum_{k} \int_{0}^{t} \int_{\Omega} G_{x_{k}}(x, y, t-s) \mu(y, s)\left(m(y, s), m_{x_{k}}(x, t)\right) d y d s \\
+ & \left.\int_{0}^{t} \int_{\Omega} G(x, y, t-s) \mu(y, s)\left(m(y, s), \Delta m_{(} x, t\right)\right) d y d s \\
= & \Delta f(x, t) .
\end{aligned}
$$

Equations (5.2)-(5.3) give

$$
\mu(x, t)+\int_{0}^{t} \int_{\Omega} G(x, y, t-s) \mu(y, s)\left(m(y, s), m_{t}(x, t)\right) d y d s
$$




$$
\begin{aligned}
& -2 \sum_{k} \int_{0}^{t} \int_{\Omega} G_{x_{k}}(x, y, t-s) \mu(y, s)\left(m(y, s), m_{x_{k}}(x, t)\right) d y d s \\
& -\int_{0}^{t} \int_{\Omega} G(x, y, t-s) \mu(y, s)(m(y, s), \Delta m(x, t)) d y d s \\
& -\int_{0}^{t} \int_{\Omega} G(x, y, t-s) a(x, t-s) \mu(y, s)(m(y, s), m(x, t)) d y d s \\
& =f_{t}-\Delta f=f^{*}(x, t) .
\end{aligned}
$$

We are going to prove the existence of solution $\mu(x, t)$ for $(5.4)$ by a fixed point argument.

Since $m(x, t)$ is given, we may assume that

$$
\begin{aligned}
\sup _{x, y \in \Omega ; s \leq t \leq T}\{|(m(y, s), \nabla m(x, t))|,|(m(y, s), \Delta m(x, t))|, \\
\left.\left|\left(m(y, s), m_{t}(x, t)\right)\right|,|a(x, t-s)(m(y, s), m(x, t))|\right\} \leq M,
\end{aligned}
$$

where $M$ is a constant. The Green's function $G(x, y, t)$ satisfies the following properties (see, e.g., [3]):

$$
\begin{gathered}
\int_{\Omega}|G(x, y, t)| d y \leq C_{1}, \quad 0<t<T, \\
\int_{\Omega}\left|\nabla_{x} G(x, y, t)\right| d y \leq \frac{C_{2}}{\sqrt{t}}, \quad 0<t<T .
\end{gathered}
$$

Here $C_{1}, C_{2}$ are constants. It is easy to see that (5.5)-(5.6) are true for the heat kernel

$$
K(x, y, t)=\frac{1}{2^{n}(\pi t)^{\frac{n}{2}}} \exp \left[-\frac{\sum\left(x_{i}-y_{i}\right)^{2}}{4 t}\right]
$$

and $K(x, y, t)$ is the leading approximation to $G(x, y, t)$ near the singularity [3].

The fixed point argument is formulated as follows:

$$
\begin{aligned}
\mu^{n+1}(x, t) & +\int_{0}^{t} \int_{\Omega} G(x, y, t-s) \mu^{n}(y, s)\left(m(y, s), m_{t}(x, t)\right) d y d s \\
& -2 \sum_{k} \int_{0}^{t} \int_{\Omega} G_{x_{k}}(x, y, t-s) \mu^{n}(y, s)\left(m(y, s), m_{x_{k}}(x, t)\right) d y d s \\
& -\int_{0}^{t} \int_{\Omega} G(x, y, t-s) \mu^{n}(y, s)(m(y, s), \Delta m(x, t)) d y d s \\
& -\int_{0}^{t} \int_{\Omega} G(x, y, t-s) a(x, t-s) \mu^{n}(y, s)(m(y, s), m(x, t)) d y d s \\
& =f_{t}-\Delta f=f^{*}(x, t) .
\end{aligned}
$$

Let

$$
A^{n}(t)=\sup \left\{\left|\mu^{n}(x, s)-\mu^{n-1}(x, s)\right| \mid x \in \Omega, 0<s<t\right\}
$$


then we have the following estimate from (5.7):

$$
A^{n+1}(t) \leq C \int_{0}^{t} \frac{1}{\sqrt{t-s}} A^{n}(s) d s+C \int_{0}^{t} A^{n}(s) d s
$$

for some constant $C$. A standard fixed point argument will then show that $\mu^{n}(x, t)$ converges uniformly and therefore we have a unique solution for (5.4).

6. Appendix B: Derivation of the second order scheme. On the righthand side of (3.39)

$$
\begin{aligned}
\frac{H}{|H|}+O\left((\Delta t)^{3}\right)=m & +\Delta t(f+\alpha \Delta m-(m, f+\alpha \Delta m) m) \\
& +(\Delta t)^{2}\left(g+\alpha \Delta f+\alpha^{2} \Delta^{2} m-(m, f+\alpha \Delta m)(f+\alpha \Delta m)\right. \\
& -\left(m, g+\alpha \Delta f+\alpha^{2} \Delta^{2} m\right) m-\frac{1}{2}|f+\alpha \Delta m|^{2} m \\
& \left.+\frac{3}{2}(m, f+\alpha \Delta m)^{2} m\right)+O\left((\Delta t)^{3}\right) .
\end{aligned}
$$

The left-hand side of (3.39) can be expanded to

$$
m_{t=t^{n+1}}=m+m_{t} \Delta t+\left.\frac{1}{2} m_{t t}(\Delta t)^{2}\right|_{t=t^{n}}+O\left((\Delta t)^{3}\right) .
$$

In order that (3.38) is satisfied, we need

$$
m_{t}=f+\alpha \Delta m-(m, f+\alpha \Delta m) m
$$

and

$$
\begin{aligned}
\frac{1}{2} m_{t t} & =g+\alpha \Delta f+\alpha^{2} \Delta^{2} m-(m, f+\alpha \Delta m)(f+\alpha \Delta m) \\
& -\left(m, g+\alpha \Delta f+\alpha^{2} \Delta^{2} m\right) m-\frac{1}{2}|f+\alpha \Delta m|^{2} m \\
& +\frac{3}{2}(m, f+\alpha \Delta m)^{2} m
\end{aligned}
$$

From (3.35), we have that

$$
\begin{aligned}
\frac{1}{2} m_{t t} & =\frac{1}{2}\left(\Delta m+|\nabla m|^{2} m\right)_{t} \\
& =\frac{1}{2} \Delta^{2} m+\frac{1}{2} \Delta\left(|\nabla m|^{2}\right) m+\nabla\left(|\nabla m|^{2}\right) \nabla m \\
& +|\nabla m|^{2} \Delta m+(\nabla m \cdot \Delta \nabla m) m+\frac{3}{2}|\nabla m|^{4} m .
\end{aligned}
$$

Equations (3.35) and (6.1) imply that

$$
f=(1-\alpha) \Delta m
$$

We will take $\alpha=\frac{1}{2}, f=\frac{1}{2} \Delta m$. Assuming $(g, m)=0$ and equating the right-hand side of (6.2) and (6.3), we have

$$
g=\nabla\left(|\nabla m|^{2}\right) \nabla m
$$


Therefore we have a second order scheme

$$
\begin{aligned}
& \frac{m^{*}-m^{n}}{\Delta t}=\frac{1}{2}\left(\Delta m^{*}+\Delta m^{n}\right)+\Delta t \nabla\left(\left|\nabla m^{n}\right|^{2}\right) \nabla m^{n}, \\
& m^{n+1}=\frac{m^{*}}{\left|m^{*}\right|} .
\end{aligned}
$$

\section{REFERENCES}

[1] K. C. Chang, W. Ding, And R. Ye, Finite-time blow-up of the heat flow of harmonic maps from surfaces, J. Differential Geom., 36 (1992), pp. 507-515.

[2] A. J. Chorin, Numerical solution of the Navier-Stokes equation, Math. Comp., 22 (1968), pp. $745-762$.

[3] A. Friedman, Partial Differential Equations of Parabolic Type, Prentice-Hall, Englewood Cliffs, NJ, 1964.

[4] B. Guo And M. C. Hong, The Landau-Lifshitz equation of the ferromagnetic spin chain and harmonic maps, Calc. Var. Partial Differential Equations, 1 (1994), pp. 311-334.

[5] O. A. Ladyzenskaya, V. A. Solonnikov, and N. N. Urelceva, Linear and quasilinear equations of parabolic type, Trans. Math. Monogr. 23, AMS, Providence, RI, 1968.

[6] M. LAKSHMANAN AND K. NAKAMURA, Landau-Lifshitz equation of ferromagnetism: Exact treatment of the Gilbert damping, Phys. Rev. Lett., 53 (1984), pp. 2497-2499.

[7] L. D. LANDAU AND E. M. Lifshitz, On the theory of the dispersion of magnetic permeability in ferromagnetic bodies, Phys. Z. Sowjetunion, 8 (1935), pp. 153-169.

[8] L. D. Landau And E. M. Lifshitz, Electrodynamics of Continuous Media, Pergamon, Oxford, 1960.

[9] K. Nakamura and T. Sasada, Soliton and wave trains in ferromagnets, Phys. Lett. A, 48 (1974), pp. 321-322.

[10] S. Ruuth, B. Merriman, J. Xin, and S. Osher, preprint.

[11] G. Strang, Accurate partial difference methods II, Numer. Math., 6 (1964), pp. 37-46.

[12] M. Struwe, Evolution problems in geometry and mathematical physics, in Nonlinear Partial Differential Equations in Differential Geometry, R. Hardt and M. Wolf, eds., IAS-Park City Math. Ser. 5, AMS, Providence, RI, 1996, pp. 257-339.

[13] P. Sulem, C. Sulem, and C. Bardos, On the continuous limit for a system of classical spins, Comm. Math. Phys., 107 (1986), pp. 431-454.

[14] S. TAN, Finite difference solutions of the Landau-Lifshitz equations with Gilbert damping term, Math. Appl., 4 (1991), pp. 35-41.

[15] A. Visintin, On Landau-Lifshitz equations for ferromagnetism, Japan J. Appl. Math., 2 (1985), pp. 69-84. 